\begin{tabular}{|c|l|}
\hline Title & In situ observation of self-organizing nanodot formation under nanosecond-pul sed Iaser irradiation on Si surface \\
\hline Author(s) & WA TA NA BE, S.; Y OSHIDA, Y.; KA Y A SHIMA, S.; Y A TSU, S.; KA W A I, M.; KA TO, T. \\
\hline Citation & $\begin{array}{l}\text { Journal of A pplied Physics, 108(10), 103510-1-103510-5 } \\
\text { https://doi.org/10.1063/13512888 }\end{array}$ \\
\hline Issue Date & 2010 \\
\hline Doc URL & http://hdl.handle.net/2115/44285 \\
\hline Rights & $\begin{array}{l}\text { Copyright 2010 A merican Institute of Physics. This article may be downloaded for personal use only. A ny other use } \\
\text { requires prior permission of the author and the A merican Institute of Physics. }\end{array}$ \\
\hline Type & article \\
\hline File Information & JAPIA U10810103510_1.pdf \\
\hline
\end{tabular}

Instructions for use 


\title{
In situ observation of self-organizing nanodot formation under nanosecond-pulsed laser irradiation on Si surface
}

\author{
S. Watanabe, ${ }^{1,2, a)}$ Y. Yoshida, ${ }^{2}$ S. Kayashima, ${ }^{2, b)}$ S. Yatsu, ${ }^{1,2}$ M. Kawai, ${ }^{3}$ and T. Kato ${ }^{4}$ \\ ${ }^{1}$ Center for Advanced Research of Energy and Materials, Faculty of Engineering, Hokkaido University, N13, \\ W8, Kita-ku, Sapporo, Hokkaido 060-8628, Japan \\ ${ }^{2}$ Division of Quantum Science and Engineering, Graduate School of Engineering, Hokkaido University, Kita- \\ 13, Nishi-8, Kita-ku, Sapporo 060-8628, Japan \\ ${ }^{3}$ High Energy Accelerator Research Organization, 1-1 Oho, Tsukuba-shi, Ibaraki-ken 305-0801, Japan \\ ${ }^{4}$ Materials Research Laboratory, Hitachi Ltd., 7-1-1 Omika, Hitachi-shi, Ibaraki-ken 319-1292, Japan
}

(Received 14 July 2010; accepted 7 October 2010; published online 19 November 2010)

\begin{abstract}
An in situ observation of the formation of a laser-irradiation-induced nanodot array on a Si surface was performed using a pulsed-laser-equipped high-voltage electron microscope (laser-HVEM). Under multiple nanosecond (ns) pulsed laser irradiation shots, atomic clusters were first formed and distributed on the surface in order to grow them epitaxially into protruded dots with diameters of ten nanometers or less. This is followed by their diffusion induced by successive laser shots to cannibalize and merge them into a ripple line with aligned, larger dots. We conclude that the present subwavelength two-dimensionally-ordered nanodot array is formed by self-organization under pulsed laser irradiation. (C) 2010 American Institute of Physics. [doi:10.1063/1.3512888]
\end{abstract}

\section{INTRODUCTION}

The irradiation of materials with an energetic beam often induces the self-organization, viz., spatiotemporal patterning, of nanostructures owing to its energy dissipative nature in a nonequilibrium open system. ${ }^{1-3}$ Well-known examples include the self-organization of lattice-defect structures induced in materials by the irradiation of energetic beams of electrons, ${ }^{4}$ neutrons, ${ }^{5,6}$ ions, ${ }^{7-9}$ and lasers. ${ }^{10-14}$ Of these, the formations of ordered dots and ripple structures on the surfaces of semiconductors by low-energy ion irradiation or by pulsed laser irradiation have attracted attention among researchers because of their potential industrial applications. For example, the two-dimensionally ordered nanodots formed on a GaSb surface by ion sputtering yield a blueshift in the photoluminescence spectrum, which is attributed to quantum confinement in the dots, and such dot-formed devices are expected to find applications in electro-optical quantum devices. ${ }^{8}$ Conical structures $^{15}$ and microcolumns ${ }^{10,14}$ on a silicon surface produced by multiple laser pulses have the potential to be used in photodetectors because of their capability to absorb incident light over a wide range of wavelengths. ${ }^{15,16}$ Similarly, the luminescent Si-nanocluster arrays fabricated by laser irradiation are also anticipated to be used in quantum dot devices. ${ }^{17,18}$

We report a laser-induced periodic surface structure of epitaxially grown dot like protrusions (hereafter, denoted as LIPSS-dots) on a $\mathrm{Si}$ surface with known regular-ripples (hereafter, denoted as conventional-LIPSS ${ }^{19-22}$ ) induced by nanosecond pulsed laser irradiation at a low laser energy density, typically less than $5 \mathrm{~kJ} / \mathrm{m}^{2}$. In addition to ex situ laser irradiation in air and in a low-pressure vessel chamber, we report how these nanodots are formed and ordered. This

\footnotetext{
${ }^{a}$ Electronic mail: sw004@eng.hokudai.ac.jp.

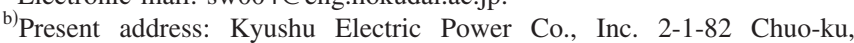
fukuoka-shi, fukuoka, Japan.
}

was investigated, for the first time, by in situ microstructural observation using a laser-equipped high-voltage electron microscope (laser-HVEM). In situ experiments, such as those using the present method of basing pulse-by-pulse under multiple laser shots by recording the microstructural evolution of a local surface region, can directly trace and reveal how the LIPSS-dots form and grow in place.

Ever since Birnbaum's discovery ${ }^{20}$ in 1965 of the grating like damage produced on semiconductor surfaces by pulsed ruby-laser irradiation, the formations of conventionalLIPSS $^{19-22}$ produced by pulsed laser irradiation on semiconductors, metals, polymers and so forth have been widely studied using various laser sources, including ultrashortpulse lasers. ${ }^{13,23-29}$ It has been well known that when a linearly polarized laser beam impinges on a material at normal incidence, the fringe pattern of conventional-LIPSS appears in the form of parallel, periodic ripple lines with an incident light wavelength separation, irrespective of the surface crystallographic orientation, and most of the damage patterns are very similar and independent of material properties, regardless of whether the material is a metal or a semiconductor. ${ }^{19}$

It is now generally accepted that conventional-LIPSS is formed by the interference between the incident laser light and the scattered waves on the surface, ${ }^{21,22}$ which may include recent laser induced waves, such as electron plasma, ${ }^{25}$ Coulomb explosion ${ }^{30}$ or surface plasmons, ${ }^{31}$ as an extension of the interference model for subwavelength (or high-spatialfrequency) LIPSS bifurcations. ${ }^{23,24,26}$ This type of model via a "top-down effect," as a natural cause of its universality involving material independence, predicts the resultant LIPSS periodicity as a linear function of the laser wavelength $\lambda$, (occasionally, with the refractive index) rather than the material surface characteristics, such as grain boundaries and crystalline orientations. An alternative explanation via a "bottom-up effect," which has been particularly proposed for subwavelength LIPSS bifurcations, is the self-organization 
originating from surface instabilities caused by two competing processes, namely, surface roughening due to explosion and surface smoothing due to self-diffusion. ${ }^{13,27}$ Moreover, Romer et al. ${ }^{29}$ were unable to account for their results of subwavelength "pre-ripple" formation, which is initiated with irregular lines and with an orientation different from that of the "regular ripples" of conventional-LIPSS induced by a number of fs-laser pulses irradiated on the surfaces of alloys, using the four previous major models in the literature. They suggested a combination of the existing models including the above-mentioned models. ${ }^{29}$

To comprehensively understand the detailed mechanisms and establish an appropriate model for such subwavelength LIPSS formation including the present LIPPS-dots, a dynamic, microscopic in situ observation under pulsed-laser irradiation on a pulse-by-pulse basis has become important.

The advantages of using HVEM for in situ observation of laser radiation, compared with other instruments, such as AFM, SEM and TEM devices, are summarized as follows. ${ }^{32}$ First, it can make the sample transparent and, thus, observable with a high spatial resolution of less than a few angstroms for a much thicker specimen than that used in conventional TEM, that is, a more realistic surface sample close to a bulky sample surface can be used. Second, HVEM has a wider specimen chamber; thus, it is suitable for in situ experimental applications. For example, although it is not a subject of the present study, a dual-beam irradiation with electrons $(>1 \mathrm{MeV})$ and laser light is also possible by our laser-HVEM.

\section{EXPERIMENT}

Single-crystal Si surfaces were irradiated ex situ with a pulsed laser radiation in air and in a low-pressure chamber $(1.3 \mathrm{~Pa})$, and in situ in laser-HVEM $\left(1.2 \times 10^{-5} \mathrm{~Pa}\right)$. We used $\mathrm{Si}(100)$ single crystalline surfaces (thickness of $400 \pm 25 \mu \mathrm{m}$ and resistivity of $22-45 \Omega \mathrm{cm}$ ). Laser irradiation with a Nd:YAG laser (Inlite II-20, Continuum: pulse repetition rate of 2-20 Hz, pulse duration of $6 \mathrm{~ns}$, central wavelength $\lambda$ of $532 \mathrm{~nm}$, linearly polarized and beam diameter of $6 \mathrm{~mm}$ ) was carried out with normal incidence to sample surfaces at an energy density of $0.2-5.0 \mathrm{~kJ} / \mathrm{m}^{2}$. The morphology of these surfaces was subsequently analyzed ex situ by scanning electron microscopy (SEM: JSM-6500, JEOL) and atomic force microscopy (AFM: VN-8000, Keyence), and the cross-sections of the ripple dots were investigated by transmission electron microscopy (TEM: JEM2010F, JEOL) with elemental analysis by x-ray energydispersive spectroscopy (EDS).

In the laser-HVEM experiment, the laser source head of the Nd:YAG pulse laser used in ex situ irradiation was mounted on to the HVEM (H-1300, Hitachi, acceleration voltage: $1300 \mathrm{kV}$, point-to-point resolution: $0.204 \mathrm{~nm}$ ) above the specimen so that the linearly polarized laser beam could enter through a quartz window and irradiate the TEM specimen at an angle of $60^{\circ}$, which corresponds to a $30^{\circ}$ (spolarized) off-beam incidence condition. Prior to laser irradiations in the laser-HVEM, the laser beam intensity profile was measured using a TEM holder with a light sensor. Im- mediately above the specimen, the laser beam passed through a 3-mm-diameter circular aperture such that the beam diameter was measured to be approximately $2 \mathrm{~mm}$ for the FWHM of the intensity. Since we did not use any optical focal lens in the present experiment, the laser intensity on the entire observing region of a TEM specimen of typically several micrometers is presumably equal. The video recording during laser irradiation was carried out at a video rate of 30 frames per second. Although the system was not sufficient for time-resolving the pulse duration, monitoring the microstructural evolution between successive pulses was achievable. The vibration effect, if any, caused by the laser shots for recording turned out to be negligible, as determined by the comparison of the sample pictures with and without laser irradiation. During in situ laser-HVEM observation, we reduced the electron intensity as much as possible to suppress electron irradiation effects and took video pictures at an under-focus-TEM condition in order to focus at the front surface part.

\section{RESULTS AND DISCUSSION}

The SEM image in Fig. 1(a) shows typical LIPSS-dots on a $\mathrm{Si}(100)$ surface, consisting of ordered dot like protrusions at a subwavelength $(<\lambda: 532 \mathrm{~nm})$. The laser irradiation was conducted in air at a pulse repetition rate of $2 \mathrm{~Hz}$ and up to 2000 pulses with an energy density of $3.5 \mathrm{~kJ} / \mathrm{m}^{2}$, normal incidence to the surfaces, and the polarization of linearly polarized light was horizontal to the images (denoted as $\boldsymbol{E})$. These peculiar, dotted ripple patterns were formed in the edge regions of a laser beam spot, where the laser energy density was relatively low (on the order of a few $\mathrm{kJ} / \mathrm{m}^{2}$ ). The periodic ripples with a wavelength separation (periodicity: $530 \mathrm{~nm}$ ) were considered to be formed by the interference between the incident beam and the light scattered from the surface, resulting in inhomogeneous energy deposition and ablation at nonuniform depths followed by rapid resolidification. $^{22}$ The AFM analysis shown in Fig. 1(b) reveals further that those LIPSS-dots, which were 50-100 nm in both height and width, sit on the top hill of the periodic ripple lines of the hill-and-valley modulation of conventional-LIPSS. Since the valley part is considered as the high-damage region such that the surface material is removed, ${ }^{33}$ the LIPSS-dots are collected into the low-damage region. Moreover, small LIPSS-dots, typically, less than a few tens of nanometers, are epitaxially grown, i.e., the protruded dots are crystalline $\mathrm{Si}$ and replicate the crystallinity of the substrate (e.g., $\mathrm{Si}\langle 100\rangle$ in Fig. 4).

As shown in Fig. 1(c), the autocorrelation functional (ACF) image analysis [shown in Fig. 1(d)] using the fast Fourier transform (FFT) for the squared part in Fig. 1(a) reveals the degree of order and the average periodicity of the arrayed dots: the dots within a ripple have a subwavelength separation of approximately $120 \pm 10 \mathrm{~nm}$, which corresponds to a separation between $1 / 5$ and $1 / 4 \lambda$, while the period of the regular ripples of aligned dots is approximately $530 \pm 25 \mathrm{~nm}$, which is close to the central wavelength of the incident laser $(\lambda: 532 \mathrm{~nm})$. The dot ordering is, however, short-ranged and weak (or loose) because only one or two 

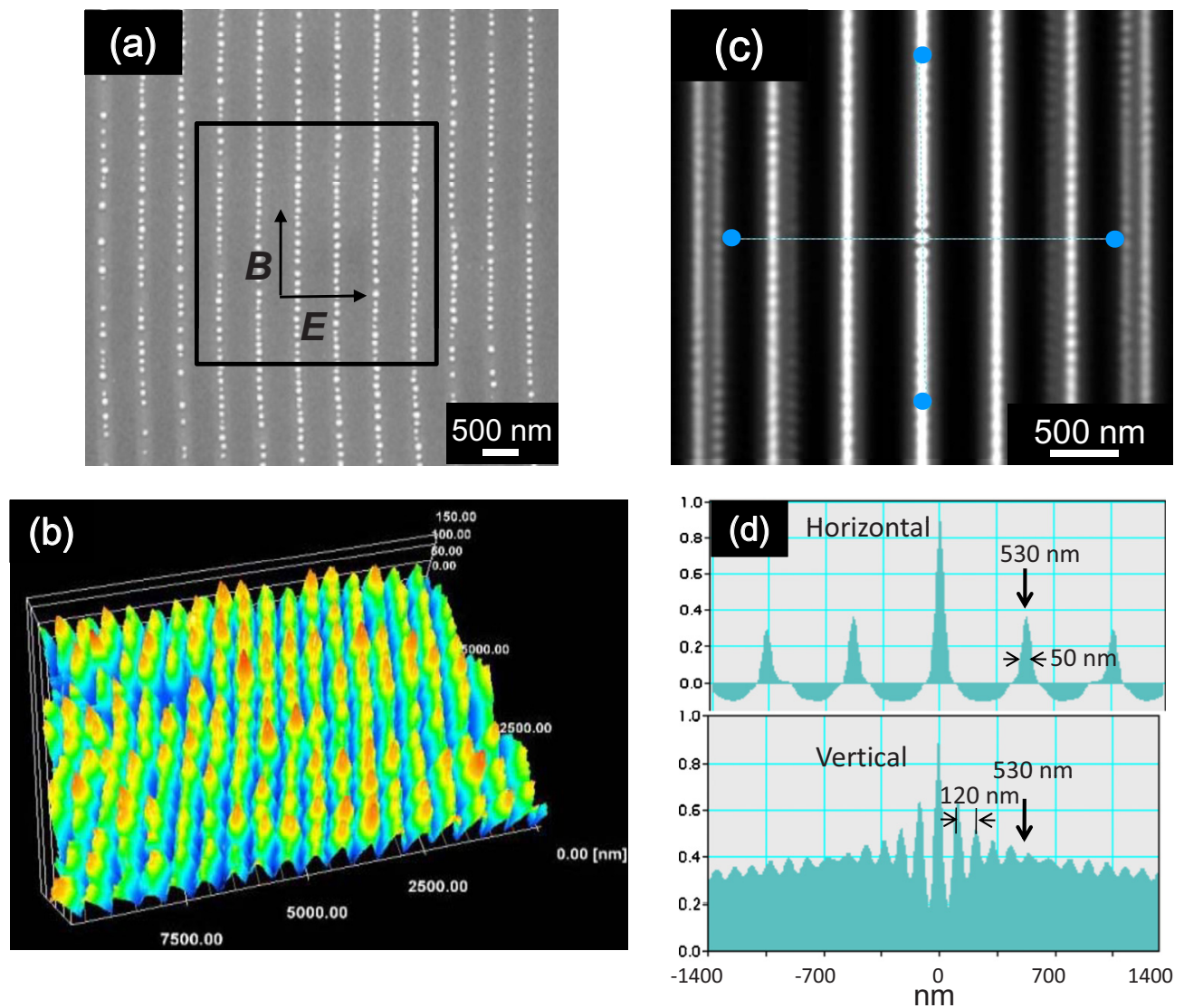

FIG. 1. (Color online) LIPPS-dots on $\mathrm{Si}(100)$ surface after $e x$ situ pulsed laser irradiation in air: (a) Ripple pattern with protruding dots formed after irradiation by 2000 laser pulses at repetition rate of $2 \mathrm{~Hz}$ and with energy density of $3.5 \mathrm{~kJ} / \mathrm{m}^{2}$. (b) Atomic force microscopy (AFM) image of LIPSS-dot ripple pattern. (c) Autocorrelation function image obtained from squared portion in (a). (d) Line profiles along horizontal (above) and vertical (below) lines in (c). The periodic distance between adjacent dots is $120 \mathrm{~nm}$ between $1 / 5$ and $1 / 4 \lambda$ of ripple line periodicity $(530 \mathrm{~nm})$ and light wavelength $(532 \mathrm{~nm})$.

nearest peaks are manifested in the vertical direction, the direction perpendicular to $\boldsymbol{E}$, [see the vertical line across the origin in Figs. 1(c) and 1(d) of the figure below] compared with that in the regular ripple lines in the horizontal direction. As shown along the far-left ripple line in Fig. 1(a), for example, no dots were observed in some places. The weakness of the dot ordering in the direction perpendicular to the polarization results in an irregular line alignment in other directions including the horizontal direction. This irregular nature might be more manifested when an additional agitation, such as a grain boundary or a diffusional force along the surface, exists.

In Fig. 2, the experimentally observed microstructural evolution and phenomenological formation of LIPSS-dots are illustrated in (a) and (b), respectively. It can be summarized for LIPSS-dot formation that by multiple laser shots, atomic clusters of small dots first form randomly on the surface (in Fig. 2, stage I/at the initial stage with 1000 pulses). In the intermediate stage, i.e., stage II (with 1500 pulses), a periodic ripple consisting of bands of smaller dots of $10 \mathrm{~nm}$ or less is formed. In stage III, the aligning stage, dots aggregate (3000 pulses) to become larger (4000 pulses). The similar clustering structures corresponding to the second stage of banded dots have been previously reported for the $\mathrm{Si}(100)$ surface induced by laser irradiation in $\mathrm{He}$ ambient ${ }^{12}$ and in vacuum, ${ }^{17}$ and the larger dots may act as a precursor of known larger bodies, such as microcolumns ${ }^{10,14}$ and cones. ${ }^{16}$
The resultant LIPSS-dots are considered to form because atomic clusters locally ablated from the surface redeposit as debris and are then irradiated again by subsequent laser
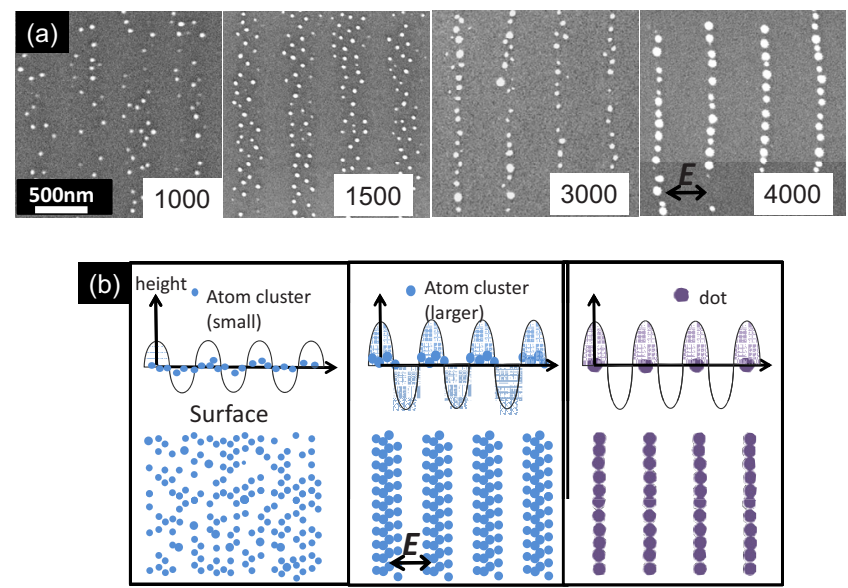

$\begin{array}{lll}\text { (I) Initial Stage (II) Intermediate Stage (III) Aligning Stage } & \end{array}$

FIG. 2. (Color online) (a) SEM images of LIPPS-dots on Si(100) surface after multiple numbers of laser shots in a low-pressure chamber $(1.33 \mathrm{~Pa})$, respectively, 1000, 1500, 3000, and 4000 (from left to right) at repetition rate of $2 \mathrm{~Hz}$ and with energy density of $1.24 \mathrm{~kJ} / \mathrm{m}^{2}$. Laser incidence was normal to surfaces and polarization was horizontal to images. (b) Schematically illustrated LIPSS-dot formation process. First stage (i): atomic cluster formation by laser irradiation with periodic surface modulation. Second (II) and third (III) stages: cluster aggregation to ripple lines and growth to larger dots due to self-organization, respectively. 

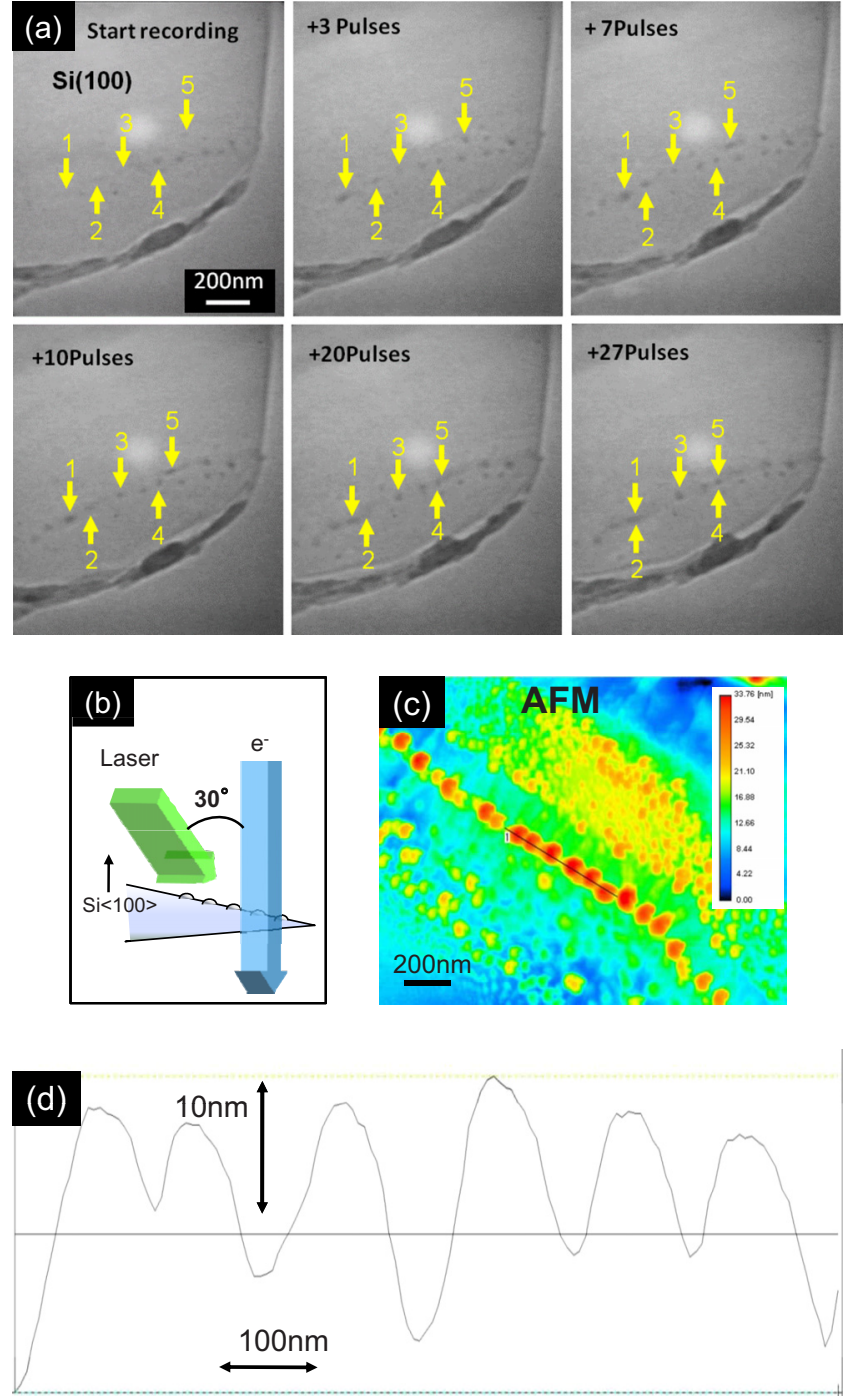

FIG. 3. (Color online) In situ laser irradiation and observation using laserHVEM $\left(1.2 \times 10^{-5} \mathrm{~Pa}\right)$ for formation and self-organization process of ordering dots on thinned TEM $\mathrm{Si}(100)$ specimen during multiple numbers of laser irradiation at repetition rate of $2 \mathrm{~Hz}$ and with energy density of $0.43 \mathrm{~kJ} / \mathrm{m}^{2}$. (a) Snapshots of dots and ripple line development on surface at certain pulse numbers recording from 30 pulses of laser irradiation using laser-HVEM. (b) Schematic illustration of plane view configuration for in situ observation using laser-HVEM (c) Height map image by AFM analysis on protruding dots and ripple pattern formation on sample surface after irradiation to 140 pulses inside laser-HVEM. (d) Height profile along the indicated line in (c). The height of the lined dots was a few tens of nanometers, and dots were separated by approximately $100-150 \mathrm{~nm}$.

shots, which results in smaller debris dots melting and inducing their aggregation into larger dot like protrusions which are accompanied by regular ripple formation. The experimental results in Fig. 2(a) indicate that the dot ordering process itself is governed by the self-organization of the cannibalization of smaller dots to yield a larger, quasi-ordered dot structure.

To put this speculation on a firm basis, we have carried out an in situ observation of the dot self-organization process in place with multiple laser shots using laser-HVEM. As shown in Fig. 3, the in situ laser-HVEM observation of LIPSS-dots developed under multiple laser pulses by irradiation on a TEM Si(100) sample surface reveals that debris like clustering dots of a few nanometers form on the surface ini-

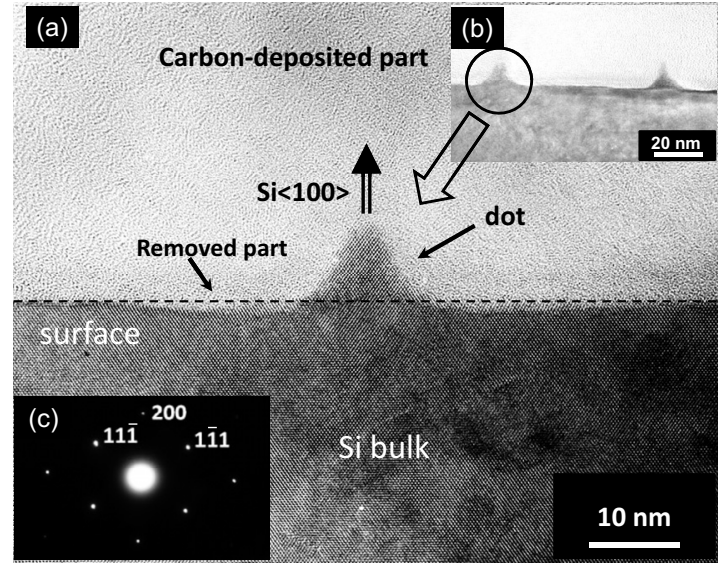

FIG. 4. High-resolution TEM (HREM) image of a small protruded dot on Si(100) surface after ex situ pulsed laser irradiation in air with 1500 laser pulses at repetition rate of $2 \mathrm{~Hz}$ and with energy density of $1.24 \mathrm{~kJ} / \mathrm{m}^{2}$. (a) The cross section HREM of the sample dot prepared along the dotted ripple line. (b) Low magnification of (a). (c) Diffraction including the dot. The HREM image in (a) (with EDS analysis: not shown) indicate that the dot was grown $\mathrm{Si}$, epitaxial to the bulk surface, surrounded by the locally removed surface portion.

tially (stage I) and that these smaller dots "diffuse" (stage II) by successive laser shots to agglomerate, producing a ripple line of aligned dots (stage III). In the snapshots from the video recording after 30 pulses shown in Fig. 3(a), as the number of laser pulses increases, dots [e.g., those indicated by numbers $1-5$ in Fig. 3(a)] form instantly on the surface (e.g., dots 1,3 , and 5 at ' +3 pulses'), and a linearly ordered array of dots develops when some of them diffuse (e.g., dots 1 and 5 move by changing their shapes) toward the line and merge into larger dots (e.g., 1 into 2 and 4 into 5 at ' +27 pluses') forming a ripple line. Such a cannibalization of smaller surface dots to larger ones, which reminds us of Ostwald ripening, ${ }^{34}$ is direct evidence of self-organization for LIPSS-dot formation, as illustrated in Fig. 2(b). The resultant, linearly-ordered dots on the surface after 140 laser pulses were shown by AFM analysis in Figs. 3(c) and 3(d) to have an upthrust morphology with a height of 10-30 nm and a separation of approximately $100-150 \mathrm{~nm}$, which are adjacent to the banded smaller dot protrusions.

The LIPSS-dots observed by both in situ and ex situ laser irradiations consist of dots aligned in ordered configurations at the subwavelength level. Our results indicate that the ordered dot like protrusions in LIPSS are formed by not only a top-down process of laser irradiation effects involving the interference between the incident and scattered waves, which predominates the regular ripple formation at a laser wavelength, but also a bottom-up process involving the selforganization of the atomic clusters generated on a surface by a laser shot, which predominates the initial stages, resulting in ordered dots at a subwavelength within the LIPSS ripples.

As shown in Fig. 4, however, the removed surface region surrounding a small dot (denoted by an arrow in Fig. 4), which is considered as an indication of uphill diffusion toward the dot top, might imply the existence of lifting force during a pulsed laser shot due to, e.g., the laser plume phenomenon via Coulomb explosion. ${ }^{35,36}$ By the limited temporal resolution of our in situ experiment, it is still unclear how 
such the smaller Si dots are generated and epitaxially grown on a substrate surface within a nanosecond laser pulse.

\section{CONCLUDING REMARKS}

Because existing models alone do not seem to explain the peculiar characteristics of the observed LIPSS-dots, a new theoretical model as well as further time-resolved experiments and a detailed analysis are also required. However, the diffusive ripening and ordering aggregation of smaller dots under laser irradiation have been directly clarified by the present in situ laser-HVEM study. The obtained results provide evidence of the self-organization mechanism in the twodimensional subwavelength ordering of the crystalline nanodots on a laser-irradiated surface, which are guided by regular ripples.

The capability of the multibeam irradiation using both laser light and electrons with laser-HVEM is expected to provide additional information on laser-electron-induced effects on the surface and inside of materials and, then, promote or renew research studies involving radiation-induced lattice defects, rapid laser annealing, and laser pluming.

\section{ACKNOWLEDGMENTS}

This work was supported in part by the Japanese Society for the Promotion of Science and the Ministry of Education, Culture, Sports, Science and Technology through the research programs of Hokkaido Innovation through Nano Technology Support (HINTS), the Collaboration Research Station of High-Voltage Electron Microscopy, and Grants-inAid for Scientific Research (A) 20246094 and (S) 19106017. We thank Professors S. Juodkazis, N. Nishiguchi, N. Sakaguchi, and T. Shibayama, and Dr. Y. Matsukawa for their helpful discussions and comments on the manuscript, and $\mathrm{Mr}$. K. Ohkubo and Mr. S. Mochizuki for their assistance with installing and operating the laser-HVEM.

${ }^{1}$ H. Qian, J. Phys. Chem. B 110, 15063 (2006)

${ }^{2}$ K. H. Li, Phys. Rep., Phys. Lett. 174, 1 (1986).

${ }^{3} \mathrm{G}$. Nicolis and I. Prigogine, Self-Organization in Nonequilibrium System: From Dissipative Structures to Order Through Fluctuations (Wiley, New York, 1977).

${ }^{4}$ K. Niwase, F. Phillipp, and A. Seeger, Jpn. J. Appl. Phys., Part 1 39, 4624 (2000).

${ }^{5}$ K. Krishan, Philos. Mag. A 45, 401 (1982).

${ }^{6}$ K. Abe, T. Masuyama, M. Satou, and M. L. Hamilton, Mater. Trans., JIM 34, 1137 (1993).
${ }^{7}$ J. Evans, Nature (London) 229, 403 (1971)

${ }^{8}$ S. Facsko, T. Dekorsy, C. Koerdt, C. Trappe, H. Kurz, A. Vogt, and H. L. Hartnagel, Science 285, 1551 (1999).

${ }^{9}$ B. Ziberi, F. Frost, B. Rauschenbach, and T. Hoche, Appl. Phys. Lett. 87, 033113 (2005).

${ }^{10}$ A. J. Pedraza, J. D. Fowlkes, and D. H. Lowndes, Appl. Phys. Lett. 74, 2322 (1999)

${ }^{11}$ T. H. Her, R. J. Finlay, C. Wu, S. Deliwala, and E. Mazur, Appl. Phys. Lett. 73, 1673 (1998).

${ }^{12}$ A. J. Pedraza, J. D. Fowlkes, and Y. F. Guan, Appl. Phys. A 77, 277 (2003).

${ }^{13}$ O. Varlamova, F. Costache, J. Reif, and M. Bestehorn, Appl. Surf. Sci. 252, 4702 (2006).

${ }^{14}$ F. Sánchez, J. L. Morenza, R. Aguiar, J. C. Delgado, and M. Varela, Appl. Phys. Lett. 69, 620 (1996).

${ }^{15}$ B. R. Tull, J. E. Carey, E. Mazur, J. P. McDonald, and S. M. Yalisove, MRS Bull. 31, 626 (2006).

${ }^{16}$ C. Wu, C. H. Crouch, L. Zhao, and E. Mazur, Appl. Phys. Lett. 81, 1999 (2002).

${ }^{17}$ B. Kumar and R. K. Soni, Nucl. Instrum. Methods Phys. Res. B 266, 2576 (2008).

${ }^{18}$ L. Patrone, D. Nelson, V. I. Safarov, M. Sentis, W. Marine, and S. Giorgio, J. Appl. Phys. 87, 3829 (2000).

${ }^{19}$ H. M. van Driel, J. E. Sipe, and J. F. Young, Phys. Rev. Lett. 49, 1955 (1982).

${ }^{20}$ M. Birnbaum, J. Appl. Phys. 36, 3688 (1965)

${ }^{21}$ J. F. Young, J. S. Preston, H. M. Vandriel, and J. E. Sipe, Phys. Rev. B 27, 1155 (1983).

${ }^{22}$ J. E. Sipe, J. F. Young, J. S. Preston, and H. M. Vandriel, Phys. Rev. B 27, 1141 (1983).

${ }^{23}$ E. M. Hsu, T. H. R. Crawford, C. Maunders, G. A. Botton, and H. K. Haugen, Appl. Phys. Lett. 92, 221112 (2008).

${ }^{24}$ A. Borowiec and H. K. Haugen, Appl. Phys. Lett. 82, 4462 (2003).

${ }^{25}$ Y. Shimotsuma, P. G. Kazansky, J. R. Qiu, and K. Hirao, Phys. Rev. Lett. 91, 247405 (2003)

${ }^{26}$ T. Q. Jia, H. X. Chen, M. Huang, F. L. Zhao, J. R. Qiu, R. X. Li, Z. Z. Xu, X. K. He, J. Zhang, and H. Kuroda, Phys. Rev. B 72, 125429 (2005).

${ }^{27}$ F. Costache, M. Henyk, and J. Reif, Appl. Surf. Sci. 208-209, 486 (2003).

${ }^{28}$ A. V. Andreev, M. M. Nazarov, I. R. Prudnikov, A. P. Shkurinov, and P. Masselin, Phys. Rev. B 69, 035403 (2004).

${ }^{29}$ G. R. B. E. Romer, A. J. H. in't Veld, J. Meijer, and M. N. W. Groenendijk, CIRP Ann. 58, 201 (2009).

${ }^{30}$ Q. H. Wu, Y. R. Ma, R. C. Fang, Y. Liao, Q. X. Yu, X. L. Chen, and K. Wang, Appl. Phys. Lett. 82, 1703 (2003).

${ }^{31}$ M. Huang, F. L. Zhao, Y. Cheng, N. S. Xu, and Z. Z. Xu, ACS Nano 3, 4062 (2009)

${ }^{32}$ T. Imura and H. Fujita, J. Electron Microsc. (Tokyo) 28, S33 (1979).

${ }^{33}$ H. Iwase, S. Kokubo, S. Juodkazis, and H. Misawa, Opt. Express 17, 4388 (2009).

${ }^{34}$ F. M. Ross, J. Tersoff, and R. M. Tromp, Microsc. Microanal. 4, 254 (1998).

${ }^{35}$ M. Khaleeq-ur-Rahman, K. Siraj, M. S. Rafique, K. A. Bhatti, A. Latif, H. Jamil, and M. Basit, Nucl. Instrum. Methods Phys. Res. B 267, 1085 (2009).

${ }^{36}$ M. Huang, F. L. Zhao, Y. Cheng, N. S. Xu, and Z. Z. Xu, Phys. Rev. B 79, 125436 (2009) 